INPLASY

PROTOCOL

To cite: Jiang et al. Effect of Aroma Inhalation Therapy on Fatigue Level: A systematic Review and Meta-analysis. Inplasy protocol 202170091. doi:

10.37766/inplasy2021.7.0091

Received: 28 July 2021

Published: 28 July 2021

Corresponding author:

Xuemei Li

Ixm_2003916@126.com

Author Affiliation:

Beijing Sport University.

Support: No support.

Review Stage at time of this submission: Formal screening of search results.

Conflicts of interest:

None declared.

\section{Effect of Aroma Inhalation Therapy on Fatigue Level: A systematic Review and Meta-analysis}

Jiang, $A^{1}$; Wang, S2; Li, X33.

Review question / Objective: The purpose of this study is to systematically evaluate the effectiveness of aromatherapy applied by inhalation on fatigue level.

Condition being studied: Fatigue is the most common problem. To cope with fatigue, complementary therapies should be used besides pharmacologic interventions. Complementary therapies included Auriculotherapy, Dry Needling, Homeopathy, Musculoskeletal Manipulations, MindBody Therapies, Naturopathy, etc. Aromatherapy is one of Mind-Body Therapies, previous studies have shown that aromatherapy can effectively control some symptoms such as fatigue, insomnia and anxiety, but the effectiveness has not yet been determined. Thus, this meta-analysis was to integrate published randomized controlled trials (RCTs) to evaluate the effectiveness of aromatherapy via inhalation on fatigue.

INPLASY registration number: This protocol was registered with the International Platform of Registered Systematic Review and Meta-Analysis Protocols (INPLASY) on 28 July 2021 and was last updated on 28 July 2021 (registration number INPLASY202170091).

\section{INTRODUCTION}

Review question / Objective: The purpose of this study is to systematically evaluate the effectiveness of aromatherapy applied by inhalation on fatigue level.

Condition being studied: Fatigue is the most common problem. To cope with fatigue, complementary therapies should be used besides pharmacologic interventions. Complementary therapies included Auriculotherapy, Dry Needling, Homeopathy, Musculoskeletal Manipulations, Mind-Body Therapies, Naturopathy, etc. Aromatherapy is one of Mind-Body Therapies, previous studies have shown that aromatherapy can 
effectively control some symptoms such as fatigue, insomnia and anxiety, but the effectiveness has not yet been determined. Thus, this meta-analysis was to integrate published randomized controlled trials (RCTs) to evaluate the effectiveness of aromatherapy via inhalation on fatigue.

\section{METHODS}

Participant or population: Participants include healthy people, patients with allergic rhinitis, hemodialysis patients, patients with knee osteoarthritis, pregnant woman, etc.

Intervention: The experimental group only received aroma inhalation therapy.

Comparator: The control group was blank control, placebo control or routine care group.

Study designs to be included: Only Included randomized controlled trials (RCTs).

Eligibility criteria: The type of literature research is randomized controlled trials. Experimental group only received aroma inhalation therapy; control group was blank control, placebo control or routine care. Measurements and results include fatigue level evaluation scale.

Information sources: Electronic databases: PubMed, EMBASE, Cochrane Library, Web of Science, CBM, CNKI, Wanfang Database, VIP. The retrieval date was January 1, 2002 to July 28, 2021.

Main outcome(s): The main outcome indicator is fatigue level evaluation scale. Include Fatigue Severity Scale, FSS; the Brief Fatigue Inventory, BFI; Postpartum Fatigue Scale, PFS; Chalder Fatigue Scale, CFS; Numerical Rating Scale, NRS; Multidimensional Assessment of Fatigue, MAF; Visual Analog Scale for Fatigue, VASF; etc.

Quality assessment / Risk of bias analysis: The risk of bias and quality of included RCTs were assessed by the Cochrane risk- of-bias tool. It includes seven specific domains: random sequence generation, allocation concealment, blinding of participants and personnel, blinding of outcome assessment, incomplete outcome data, selective outcome reporting and other sources of bias.

Strategy of data synthesis: STATA statistical software $\mathbf{1 6 . 0}$ was used for metaanalysis. Standardized mean differences (SMD) and 95\% confidence interval (CI) were calculated. The heterogeneity among selected studies was assessed using the $\mathrm{I}^{2}$. $\mathbf{l}^{2}<50 \%, \mathbf{P}>0.05$ was considered as low heterogeneity, and apply fixed model; otherwise, a random-effect model will be used when $I^{2} \geq 50 \%, P \leq 0.05$. Egger's test and Begg's funnel plot were used to assess potential publication bias.

Subgroup analysis: If the heterogeneity is significant, we will conduct a subgroup analysis. The different characteristics, different characteristics of participants, duration of treatment and different treatment types were classified and compared to determine the source of heterogeneity.

Sensitivity analysis: Another method to determine the source of heterogeneity is sensitivity analysis, which can make the experimental results more reliable and stable.

Country(ies) involved: China.

Keywords: Aromatherapy; Inhalation; Fatigue; Meta-analysis.

Contributions of each author:

Author 1 - Aiyun Jiang.

Email: irene0340@126.com

Author 2 - Siqi Wang.

Author 3 - Xuemei Li

Email: Ixm_2003916@126.com 